\title{
Fuzzy Modeling of Reoccupation of a Leaf-cutting Ants' Trail
}

\author{
R.S.M. JAFELICE and C.G. ALMEIDA* \\ Received on November 29, 2013 / Accepted on January 27, 2015
}

\begin{abstract}
In this paper we model the reoccupation of a trail by leaf-cutting ants. The mathematical modeling consists in studying the leaf-cutting ants' displacement towards an attraction area and their return to the nest. To carry out this research, we utilized the diffusion-advection equation, where the dispersion and velocity towards $x$ and $y$ are fuzzy parameters. The experts affirm that the pheromone is an important variable concerning trail reoccupation because it influences leaf-cutting ants dispersion and velocity of displacement. The numerical simulations took into account the pheromone through the Fuzzy Rule-Based System (FRBS). The numerical results are compatible with the biological phenomenon studied.
\end{abstract}

Keywords: fuzzy parameters, partial differential equations, leaf-cutting ants.

\section{INTRODUCTION}

The study of the behavior of ants' colonies, along with the study of their displacement towards foraging trails are, nowadays, not only restricted to biologists' interest. Researchers from different fields of study such as engineering, applied mathematics and computer science are also interested in the field known as Artificial Intelligence [2]. The understanding of the dynamics ruling ants' displacement in a foraging trail might be useful not only to elaborate algorithms that are based in artificial intelligence, but also to contribute in planning efficient control against ants in agricultural regions. Upon the mathematical view, the complexity of this dynamical can be modeled through partial differential equations with fuzzy parameters.

The objective of this research is to model leaf-cutting ants' reoccupation in a specific trail. The authors present a differentiated study regarding results published previously [3], [4] and [5]. The novelty is that the biological modeling considers both ants' displacement from the nest towards the attraction region and their return to the nest. Another important modeling characteristic is the introduction of the pheromone as linguistic variable input of the FRBS. The FRBS outputs generate the partial differential equation parameters that rule the phenomenon studied: dispersion and velocity in both horizontal and vertical directions.

*Corresponding author: César Guilherme Almeida

Faculdade de Matemática, UFU - Universidade Federal de Uberlândia, 38408-144 Uberlândia, MG, Brasil.

E-mails: rmotta@ufu.br; cesargui@ufu.br; cesar_ga@sbmac.org.br 
The biological characteristics presented in the model are based in research of Vasconcelos [7] carried out in Amazon Forest with a colony of Atta cephalotes, known as leaf-cutting ants. His research was conducted from July 1985 to January 1986 and from September 1986 to March 1987 in which trails were determined as shown in Figure 1, where the broken line represents the foraging range and the continuous line represents foraging territory that was effectively occupied by the ants. Solid triangles represent plants that were attacked by the ants in the period of July 1985 and January 1986, empty triangles represent plants that were attacked by ants in the period of September 1986 and March 1987 and solid squares represent plants that were attacked in both periods. Finally, the dotted lines represent trails radiating from the nest (shaded area). The calculation of the dispersion was based upon information from the traced polygonal region $(\mathrm{P})$ whereas the computational simulations took into account the rectangular region $(\mathrm{R})$ (both regions are highlighted in Fig. 1). More details about the calculations and elaborations of the FRBS input and output variables membership functions can be found in [5]. In the next session we will present the mathematical model studied.

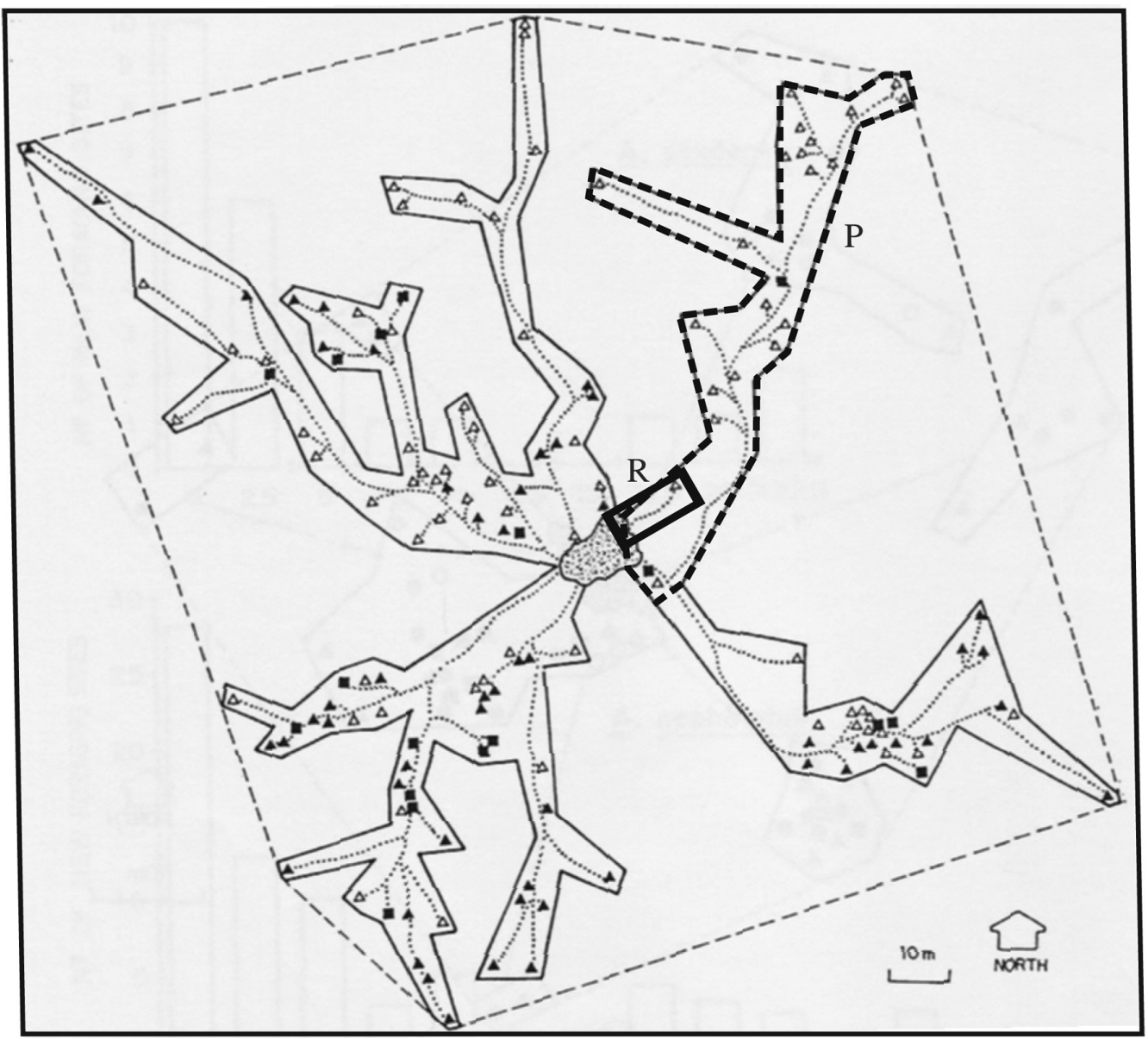

Figure 1: Trails and foraging territory [7]. 


\section{FUZZY MODEL}

The proposed model for the trail reoccupation by leaf-cutting ants is given by the differential equation:

$$
\frac{\partial P}{\partial t}+v(l o c, s) \cdot \nabla P-\nabla \cdot\left(\alpha\left(P, l_{\text {loc }}, s\right) \nabla P\right)=0 .
$$

In the equation (2.1) the variable $P=P(\mathbf{x}, t)$ indicates the population in instant $t \in[0, T]$ and in position $\mathbf{x}$ belonging to domain $\Omega \subset \mathbf{R}^{2}$ (Fig. 2). We assumed that population dispersion is represented by parameter $\alpha\left(P, l o c_{t o t}, s\right)$, where total locomotion $\left(l o c_{t o t}\right)$ is determined by a FRBS that depends on the input variables $l o c_{x}$ and $l o c_{y} ; s$ is the chemical signal represented by the pheromone left behind by ants in the area. Velocity of population displacement is $v(l o c, s)=$ $\left(v_{1}\left(l o c_{y}, s\right), v_{2}\left(l o c_{x}, s\right)\right)$, where $l o c$ is the vectorial function, $l o c=\left(l o c_{x}, l o c_{y}\right)$, which supplies values for locomotion along directions $x$ and $y$. In this way, velocity depends on pheromone and subregions of domain, which indicate ants' difficulties in locomotion on the terrain. The linguistics variables of the locomotion parameters took into account the subregions represented in Figure 2. The pheromone linguistic variable was represented by three linguistic terms: weak, strong and very strong (Fig. 9). In the attraction region and in the nest, the pheromone was classified as very strong, on the trails as strong and in other domain regions as weak.

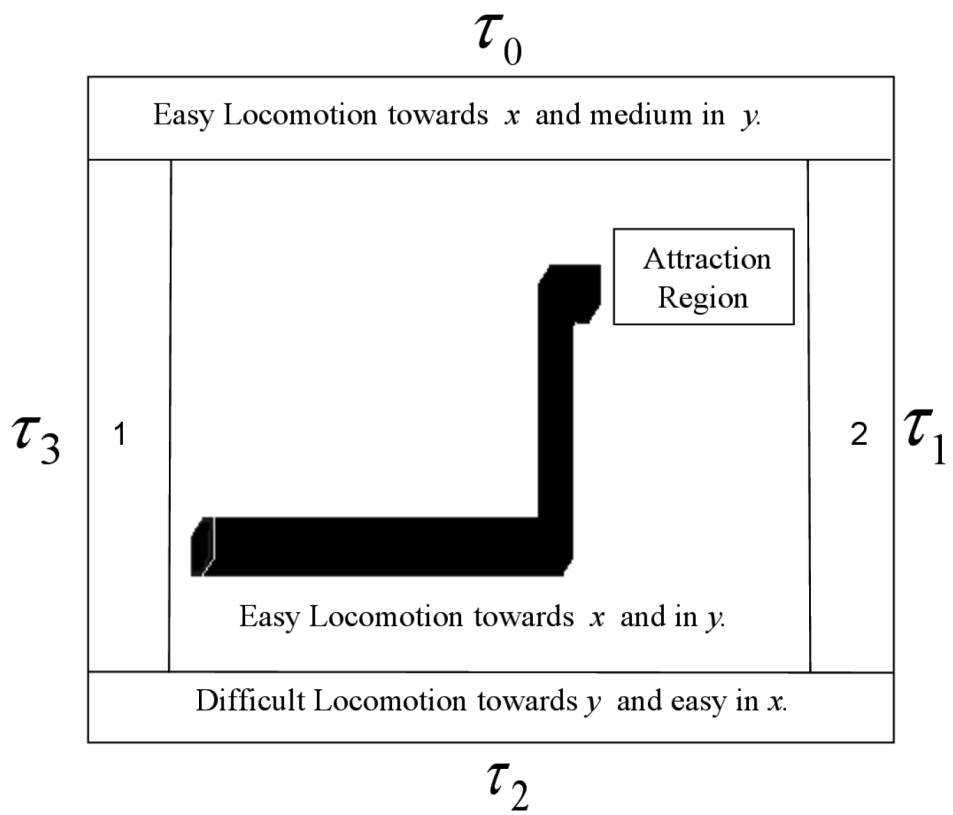

1 - Difficult Locomotion towards $x$ and in $y$.

2 - Easy Locomotion towards $y$ and difficult in $x$.

Initial condition

Figure 2: Domain $\Omega$. 
The boundary conditions are given as follows:

$$
\begin{array}{lll}
\alpha\left(P, l_{\text {loc }}, s\right) \frac{\partial P}{\partial y}=P v_{2}\left(l o c_{x}, s\right), & \forall \mathbf{x} \in \tau_{0}, & \forall t \in[0, T] \\
\alpha\left(P, \text { loc }_{t o t}, s\right) \frac{\partial P}{\partial x}=P v_{1}\left(l o c_{y}, s\right), & \forall \mathbf{x} \in \tau_{1}, & \forall t \in[0, T] \\
\alpha\left(P, \text { loc }_{t o t}, s\right) \frac{\partial P}{\partial y}=P v_{2}\left(l o c_{y}, s\right), & \forall \mathbf{x} \in \tau_{2}, & \forall t \in[0, T] \\
\alpha\left(P, \text { loc }_{t o t}, s\right) \frac{\partial P}{\partial x}=P v_{1}\left(l o c_{y}, s\right), & \forall \mathbf{x} \in \tau_{3}, & \forall t \in[0, T] .
\end{array}
$$

The initial condition is given by:

$$
P(\mathbf{x}, 0)=P_{0}(\mathbf{x}), \quad \forall \mathbf{x} \in \Omega .
$$

The initial condition $P_{0}(\mathbf{x})$ is distributed along the trail as shown in Figure 2. The nest is modeled utilizing a square region that contains 25 ants in each one of its vertices (nodes of first-order finite elements). In each nest node of the trail, $10 \%$ of the population was placed.

In order to find the numerical solution of equation (2.1) with boundary conditions (2.2)-(2.5); and initial condition (2.6), the finite element method was used with a uniform triangular grid and linear functions [6]. As for the discretization in time the Crank-Nicolson method was used [1].

In Figure 3 we present the scheme for dispersion calculation that utilizes the FRBS 1 and FRBS 2. The FRBS 1 input linguistic variables are the locomotion towards $x$ and $y$; the output variable is the total locomotion. The FRBS 1 output variable will be one of the input linguistic of FRBS 2 variables along with the variables: population and pheromone. As the output FRBS 2 depends on the population, which is a time function, the dispersion should be evaluated in each step of time of the computational program (software Matlab).

In Figure 4 we present scheme of the velocity calculations towards $x$ and $y$. Velocity towards $y$ is the output variable FRBS 3 that considers the input linguistic variable as the locomotion towards $x$ and pheromone. In the FRBS 4 the output variable is velocity towards $x$ and the input linguistic variables are the locomotion towards $y$ and pheromone.

Membership functions of all linguistic variables are trapezoidal, except the pheromone functions that are characterized by unitary fuzzy set or singleton (Fig. 9). The singleton format of such functions was adopted with the aim of adaptation to the biologic phenomenon, since the pheromone or chemical signal is strongly concentrated on the trails [2]. Figures 5 and 6 present membership functions towards $y$ and total locomotion. Note that both linguistic variables are defined in the same interval, from 0 to 1 , and are expressed by linguistic terms: difficult, medium and easy. The same treatment is given to locomotion linguistic variable towards $x$.

The population which is one of the FRBS 2 input variable has the membership functions as presented in Figure 7. This variable is defined in the interval from 0 to 100 and their linguistic terms are: small, medium and large. The FRBS 2 output variable has four linguistic terms which are: very low, low, medium and high and it is defined from 0 to 0.14 as depicted in Figure 8. 


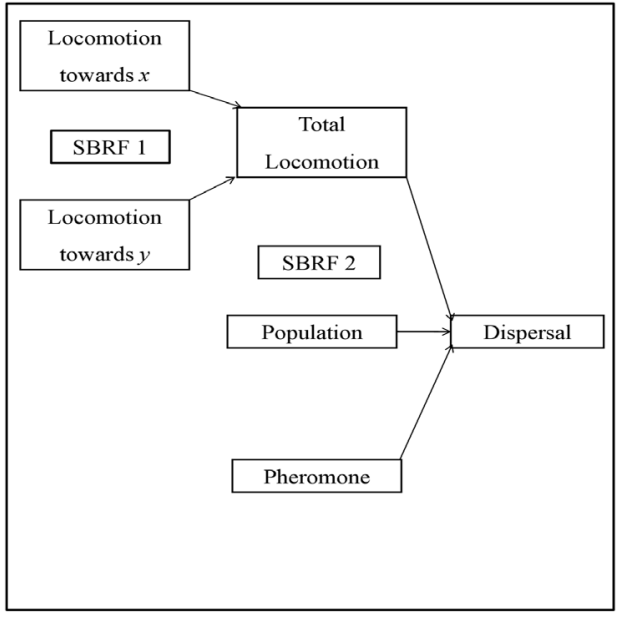

Figure 3: Scheme calculation of dispersion through the FRBS.

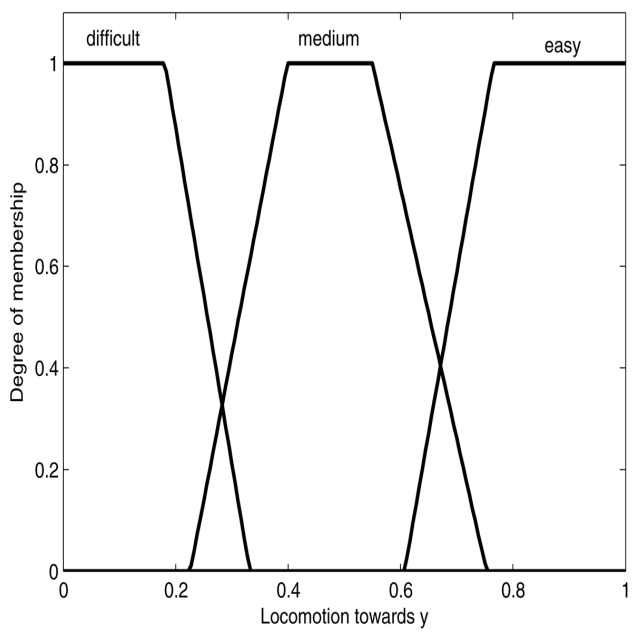

Figure 5: Membership functions for locomotion towards $y\left(l o c_{y}\right)$.

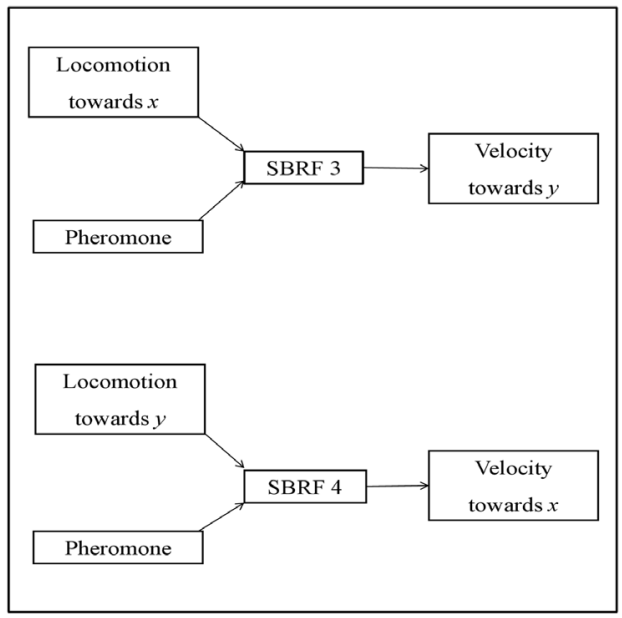

Figure 4: Scheme of the velocity calculation through the FRBS.

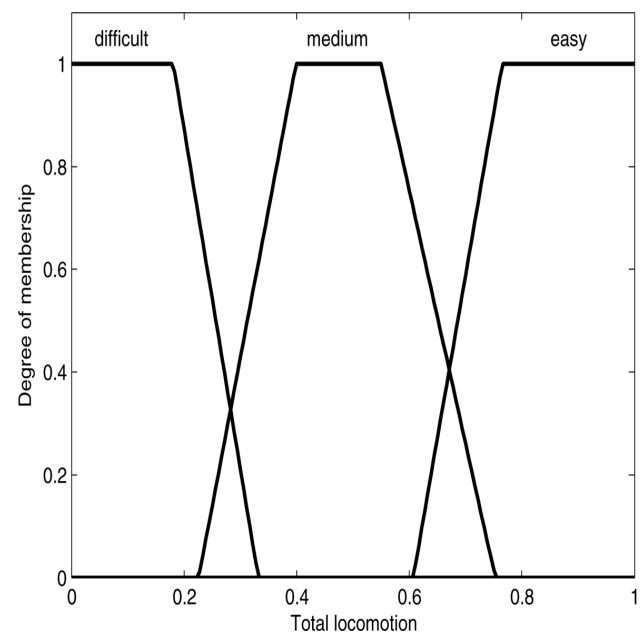

Figure 6: Membership functions for total locomotion $\left(\right.$ loc $\left._{t o t}\right)$.

Figure 10 presents velocity membership functions in direction $x$. Note that the linguistic variable is defined in the interval from 0 to 0.8 and is expressed by the linguistic terms: low, medium and high. The velocity linguistic variable towards $y$ is expressed similarly and is defined in the interval from 0.01 to 0.05 .

Fuzzy base rules were built with the support of the biologist Prof. Dr. H.L. Vasconcelos. Following, we will present a fuzzy rule for each one of the FRBS: 


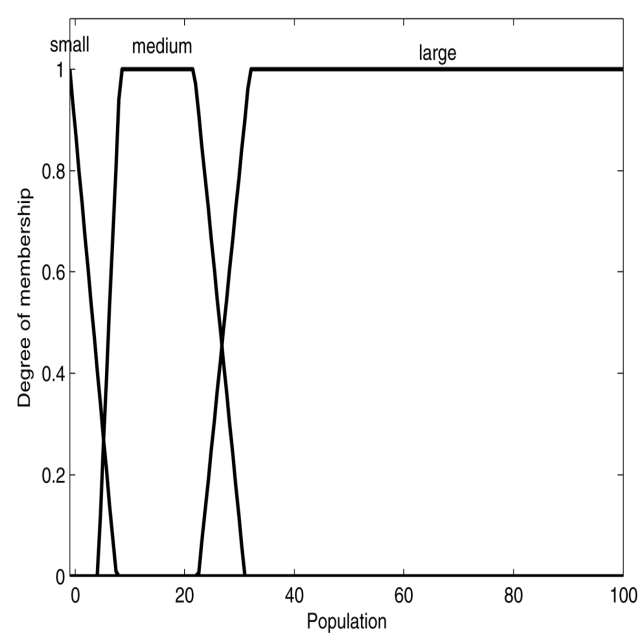

Figure 7: Membership functions for population $(P)$.

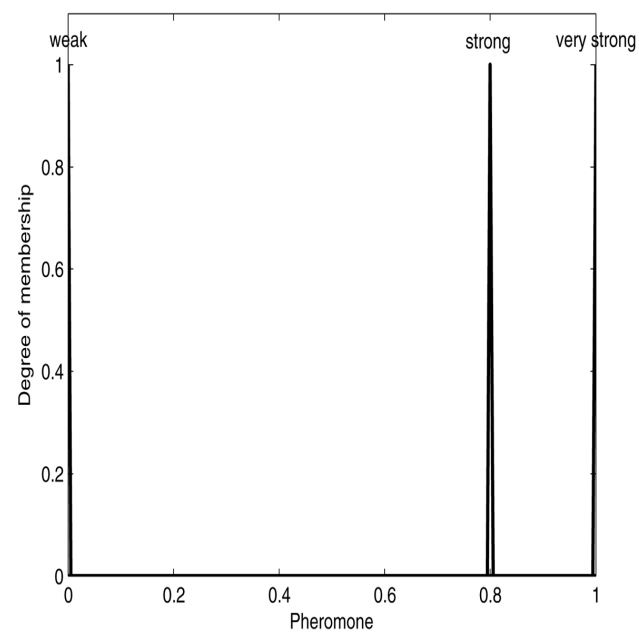

Figure 9: Membership functions for pheromone $(s)$.

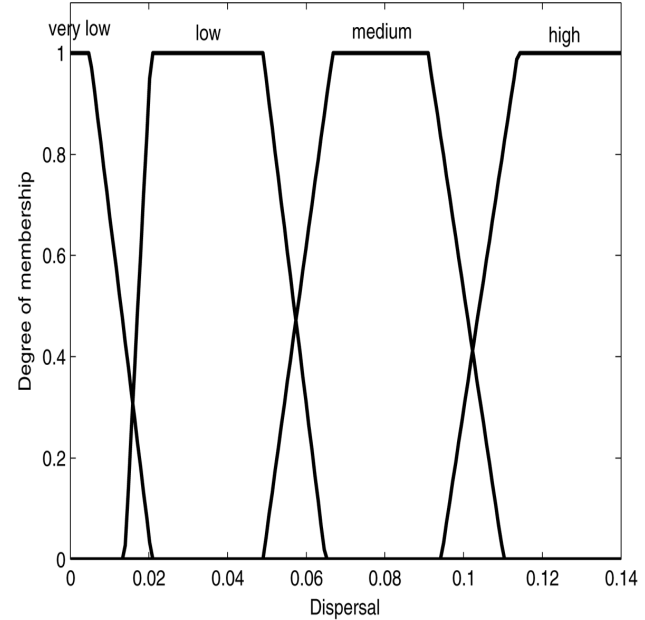

Figure 8: Membership functions for dispersal $(\alpha)$.

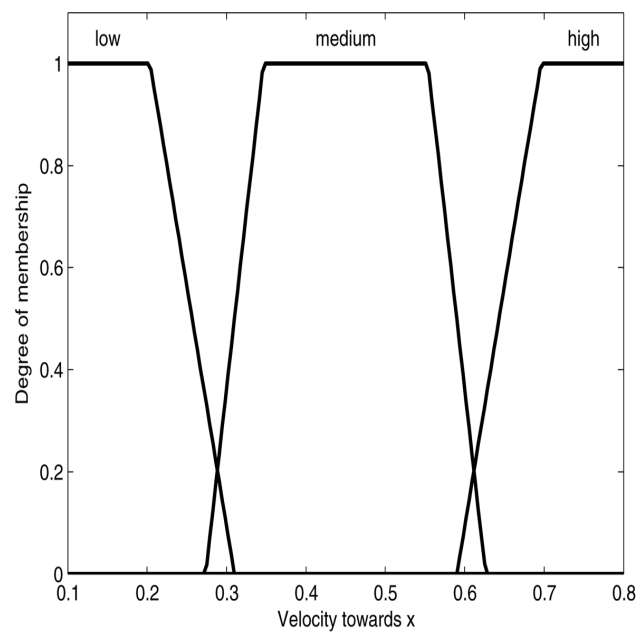

Figure 10: Membership functions for velocity towards $x\left(v_{1}\right)$.

- The FRBS 1 has 9 fuzzy rules; example of those rules:

If $l o c_{x}$ is easy and $l o c_{y}$ is difficult then $l o c_{t o t}$ is medium.

- The FRBS 2 has 27 fuzzy rules; example:

If $P$ is large and $l o c_{t o t}$ is medium and $s$ is strong then $\alpha$ is low.

- The FRBS 3 and FRBS 4 have 9 similar fuzzy rules; example:

If $l o c_{x}$ is easy and $s$ is strong then $v_{2}$ is high. 


\section{RESULTS}

Simulations are accomplished considering a $6 \mathrm{~m}$ long by $3 \mathrm{~m}$ wide rectangular domain $\Omega$ (Fig. 1). Ants' displacement from the nest to the attraction region is simulated considering a time interval of 4 hours (see Fig. 11). The nest is represented by 100 ants uniformly distributed on the nodes of a square region composed of two finite triangular elements. The attraction region is composed of eight finite elements. Displacement from the attraction region towards the nest is simulated with a time interval of 2 hours (see Fig. 12). Observe that the initial condition of returning displacement is given by the Figure 11, that represents final distribution of the population displacement regarding leaving the nest towards the attraction region.

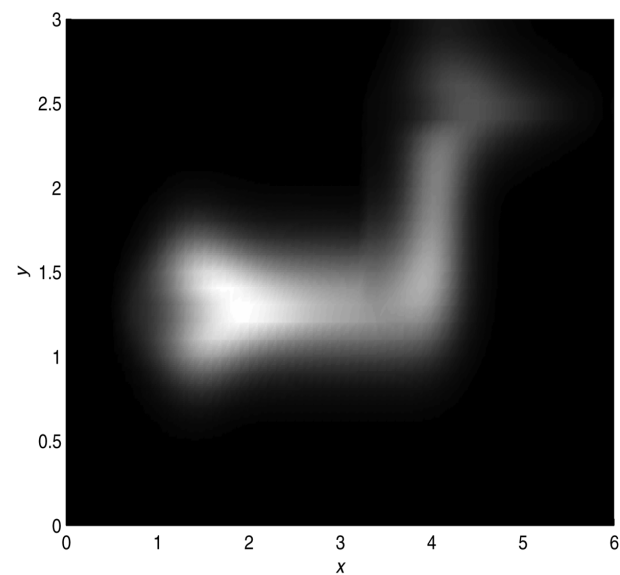

Figure 11: Displacement of ants from the nest to the attraction region.

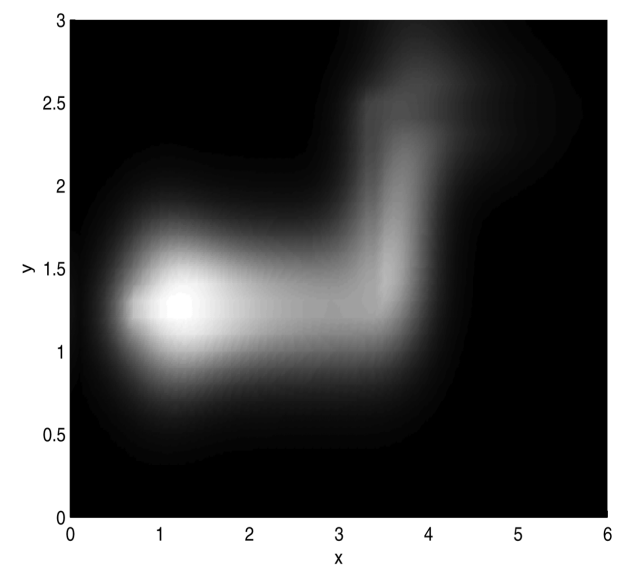

Figure 12: Displacement of ants from the attraction region to the nest.

The graphs exhibited in Figure 13 and 14 represent the quantity of ants in a node in the nest and in a node on attraction region, respectively, considering ants' displacement from the nest to the attraction region. Results presented are consistent with the biological phenomenon observed, as follows: the occurrence of a decrease of the number of ants in the nodes of the nest as well as an increase of the number of ants in the node at the attraction region. The graphs in Figures 15 and 16 are related to ants' displacement from the attraction region to the nest and represent also the quantity of ants in one nest node and in one node in the attraction region. In this case, results presented are also consistent with the biological phenomenon observed: the occurrence of a decrease of the amount of ants in the node of the nest and a decrease in the node of the attraction region.

\section{CONCLUSION AND FUTURE WORK}

The use of fuzzy modeling made it possible to incorporate in this research complex questions regarding the presence of pheromone on the reoccupation of a trail, locomotion difficulties on the terrain and the influence of the amount of ants in the parameter providing dispersion of 


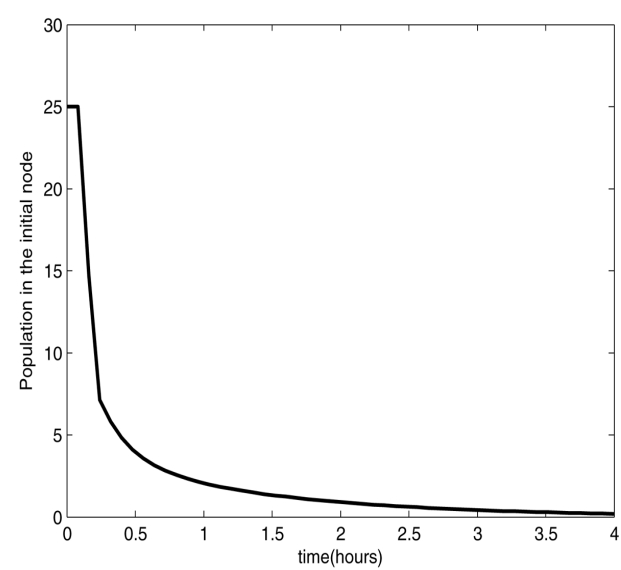

Figure 13: Quantity of ants in the node of the nest (leaving the nest).

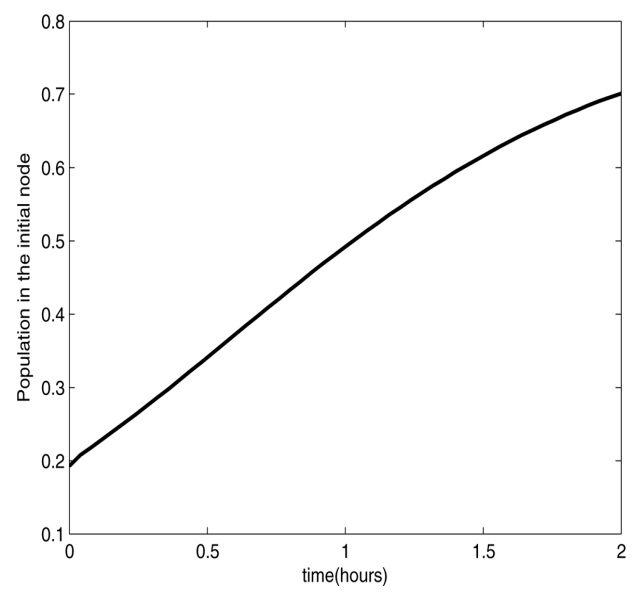

Figure 15: Quantity of ants in the node of the nest (return).

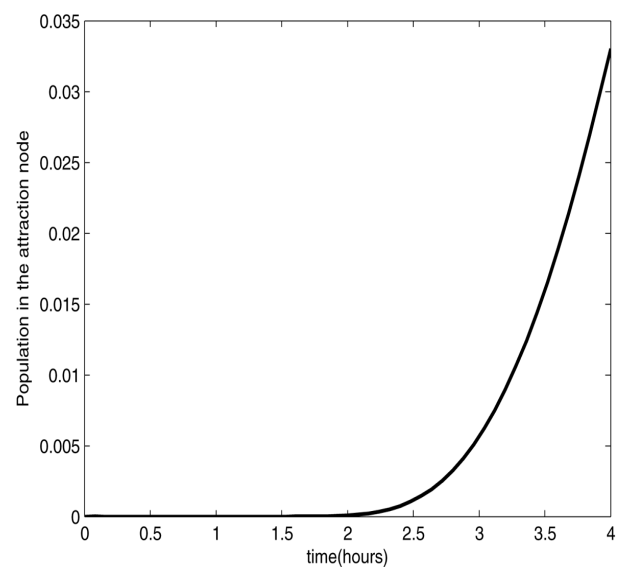

Figure 14: Quantity of ants in the node of the attraction region (leaving the nest).

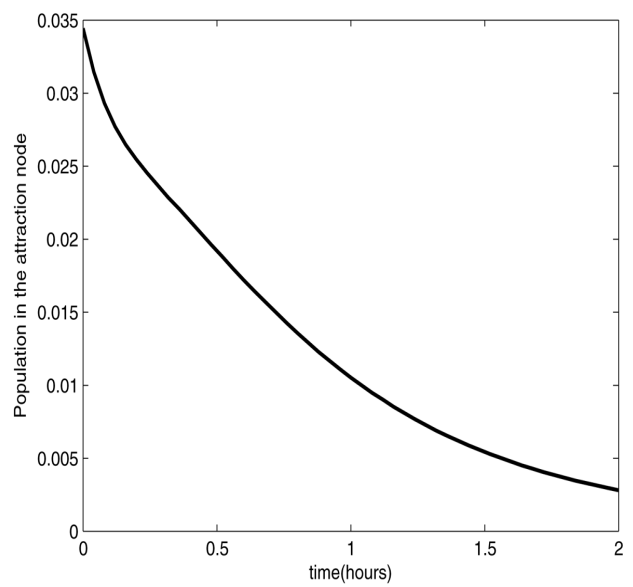

Figure 16: Quantity of ants in one node of the attraction region (return).

a diffusion-advection differential equation. The linguistic variable "pheromone" was built taking into account the fact that ants while moving, deposit on the ground a substance called pheromone. The reoccupation of the trail was modeled considering that ants possess certain tendency to follow the pheromone left on the trails, and regions less visited present small quantities of pheromone [2].

There were some difficulties to adjust computationally all the parameters involved in this modeling. Some of those parameters were obtained from biological information while others were chosen empirically but allowing satisfactory results. They were presented in the previous section. 
An improvement in the model is being elaborated with the inclusion of one more attraction region and consequently the displacement of ants towards a new trail.

All the techniques developed to investigate this specific study on ants can be applied to other studies which are modeled with analogous equations. For instance, the problem of incompressible miscible displacement in porous media, the parameters of the dispersion-diffusion tensor (molecular diffusion; longitudinal dispersion and transversal dispersion) can be treated as fuzzy parameters.

RESUMO. Neste trabalho, nós modelamos a reocupação de uma trilha de formigas cortadeiras. A modelagem matemática consiste em estudar o deslocamento de formigas cortadeiras em direção a uma região de atração e o retorno das mesmas para o formigueiro. Para conduzir esta pesquisa, nós utilizamos uma equação de difusão-convecção, na qual a dispersão e a velocidade nas direções horizontal $(x)$ e vertical $(y)$ são parâmetros fuzzy. Os especialistas afirmam que o feromônio é uma importante variável no que se refere à reocupação de uma trilha porque o mesmo pode interferir no comportamento das formigas cortadeiras, ocasionando alterações na dispersão e na velocidade do deslocamento. As simulações numéricas levaram em consideração o feromônio através de Sistemas Baseados em Regras Fuzzy (SBRF). Os resultados numéricos são compatíveis com o fenômeno biológico estudado.

Palavras-chave: parâmetros fuzzy, equações diferenciais parciais, formigas cortadeiras.

\section{REFERENCES}

[1] R.S. Cantrell \& C. Cosner. Spatial Ecology via Reaction-Diffusion Equations. John Wiley and Sons Ltd., England (2003).

[2] M. Dorigo \& T. Stützle. Ant Colony Optimization, Massachusetts Institute of Tecnology, USA (2004).

[3] R.M. Jafelice, C.G. Almeida, J.F.C.A. Meyer \& H.L. Vasconcelos. Dispersal of Leaf-Cutting Ants: Fuzzy Mathematical Modeling, Numerical Approximation and Simulations. In: IFSA Word Congress - EUSFLAT Conference, Lisboa, (2009), 271-276.

[4] R.M. Jafelice, C.G. Almeida, J.F.C.A. Meyer \& H.L. Vasconcelos. A Fuzzy-parameter Dispersionattraction Partial Differential Model for an Ant Colony. In: XXXII Congresso Nacional de Matemática Aplicada e Computacional, Cuiabá, (2009), 87-93.

[5] R.M. Jafelice, C.G. Almeida, J.F.C.A. Meyer \& H.L. Vasconcelos. Fuzzy parameters in a partial differential equation model for population dispersal of leaf-cutting ants. Nonlinear Analysis: Real World Applications, 12 (2011), 3397-3412.

[6] H. Kardestuncer \& D.H. Norrie. Finite Elements Handbook, McGraw-Hill, New York (1987).

[7] H.L. Vasconcelos. Foraging activity of two species of leaf-cutting ants (Atta) in a primary forest of the central Amazon. Insectes Sociaux, 37(2) (1990), 131-145. 